\title{
BMJ Open Effectiveness and safety of nicotine patches combined with e-cigarettes (with and without nicotine) for smoking cessation: study protocol for a randomised controlled trial
}

\author{
Natalie Walker, ${ }^{1}$ Marjolein Verbiest, ${ }^{1,2}$ Tomasz Kurdziel, ${ }^{1}$ George Laking, ${ }^{3}$ \\ Murray Laugesen, ${ }^{4}$ Varsha Parag, ${ }^{1}$ Chris Bullen ${ }^{1}$
}

To cite: Walker $\mathrm{N}$, Verbiest $\mathrm{M}$, Kurdziel T, et al. Effectiveness and safety of nicotine patches combined with e-cigarettes (with and without nicotine) for smoking cessation: study protocol for a randomised controlled trial. BMJ Open 2019;9:e023659. doi:10.1136/ bmjopen-2018-023659

\section{- Prepublication history for} this paper is available online. To view these files, please visit the journal online (http://dx.doi. org/10.1136/bmjopen-2018023659).

Received 19 April 2018 Revised 15 October 2018 Accepted 20 December 2018

Check for updates

(c) Author(s) (or their employer(s)) 2019. Re-use permitted under CC BY-NC. No commercial re-use. See rights and permissions. Published by BMJ.

${ }^{1}$ School of Population Health, National Institute for Health Innovation, The University of Auckland, Auckland, New Zealand

${ }^{2}$ Tilburg University, Tilburg, The Netherlands

${ }^{3}$ Department of Oncology, School of Medical Sciences, The University of Auckland, Auckland, New Zealand ${ }^{4}$ Department of Psychology, University of Canterbury, Christchurch, New Zealand

Correspondence to

Dr Natalie Walker;

n.walker@auckland.ac.nz

\section{ABSTRACT}

Introduction Evidence indicates e-cigarettes can help people quit smoking; however, more confirmatory trials are needed. To date, no trials have evaluated the effectiveness and safety of combining nicotine patches with e-cigarettes (with and without nicotine) for smoking cessation.

Methods and analysis This study is a pragmatic, three-arm, community-based, single-blind, randomised trial undertaken in New Zealand. Eligible participants are daily/non-daily smokers, aged $\geq 18$ years, naive e-cigarette users and motivated to quit smoking in the next 2 weeks. Participants $(n=1809)$, recruited using multi-media advertising, are randomised to 14 weeks of (1) $21 \mathrm{mg}$ nicotine patches ( $\mathrm{n}=201)$; (2) $21 \mathrm{mg}$ nicotine patches $+18 \mathrm{mg} / \mathrm{mL}$ nicotine e-cigarette $(\mathrm{n}=804)$; or (3) $21 \mathrm{mg}$ nicotine patches+nicotine free e-cigarette $(n=804)$. Participants receive weekly withdrawal-oriented behavioural support calls for 6 weeks post-randomisation. The primary outcome is self-reported biochemically verified continuous abstinence (CA) at 6 months post quit-date. The primary comparison is nicotine patch + nicotine e-cigarette versus nicotine patch + nicotine free e-cigarette, and the secondary comparison is nicotine patch versus nicotine patch +nicotine e-cigarette $(90 \%$ power, $p=0.05$, to detect an absolute difference in 6 month CA rates of $8 \%$ and $15 \%$ respectively). Secondary outcomes, collected by phone interview at quit date, then $1,3,6$ and 12 months post-quit date, include self-reported CA, 7 day point prevalence abstinence, cigarettes per day (if smoking, or when smoking for non-daily smokers), time to relapse (if returned to smoking), belief in ability to quit, use of other cessation support, side effects/ serious adverse events, treatment compliance, seeking additional support around e-cigarette use, daily use of both e-cigarettes and cigarettes, use of treatment past 14 weeks, views on treatment and recommendation to others, weight and cost-per-quitter.

Ethics and dissemination The Northern A Health and Disability Ethics Committee approved the trial. Findings will be disseminated through publication, conference/meeting presentations, and media.

Trial registration number NCT02521662; Pre-results.
Strengths and limitations of this study

- This is the first trial to investigate the effectiveness and safety of combining nicotine patches and e-cigarettes on smoking abstinence.

- This is the first large community-based trial testing a second generation e-cigarette for smoking cessation, with choice of device and juice undertaken in consultation with members of the vaping industry.

- The trial is undertaken in a country with strong tobacco control measures in place, and low uptake of e-cigarettes.

- The trial is pragmatic in design, with open eligibility and no participant payments, enabling greater generalisability.

- For ethical reasons, in New Zealand it was not possible to include a fourth comparison group of placebo patches.

\section{INTRODUCTION}

Smoking cessation treatments should address at least two aspects of tobacco dependence: physiological and behavioural dependence. ${ }^{1}$ Although nicotine replacement therapies (NRT) help address the physiological dependence of cigarette smoking by providing nicotine to the body, they don't mimic the habituated tactile behaviours (involving the mouth and hands) associated with cigarette use. $^{2}$

Electronic cigarettes (e-cigarettes) have considerable potential to help people quit smoking as they address both the physiological and behavioural dependence of tobacco smoking. ${ }^{34}$ These devices deliver nicotine by a form of aerosolisation (popularly known as vaping) and are likely safer to use than smoking tobacco as users have reduced exposure to tobacco toxicants. ${ }^{5-10}$ To date, only two randomised trials with 6 month abstinence 
Table 1 Summary of the design and outcomes from the two published trials of e-cigarettes for 6 month smoking cessation

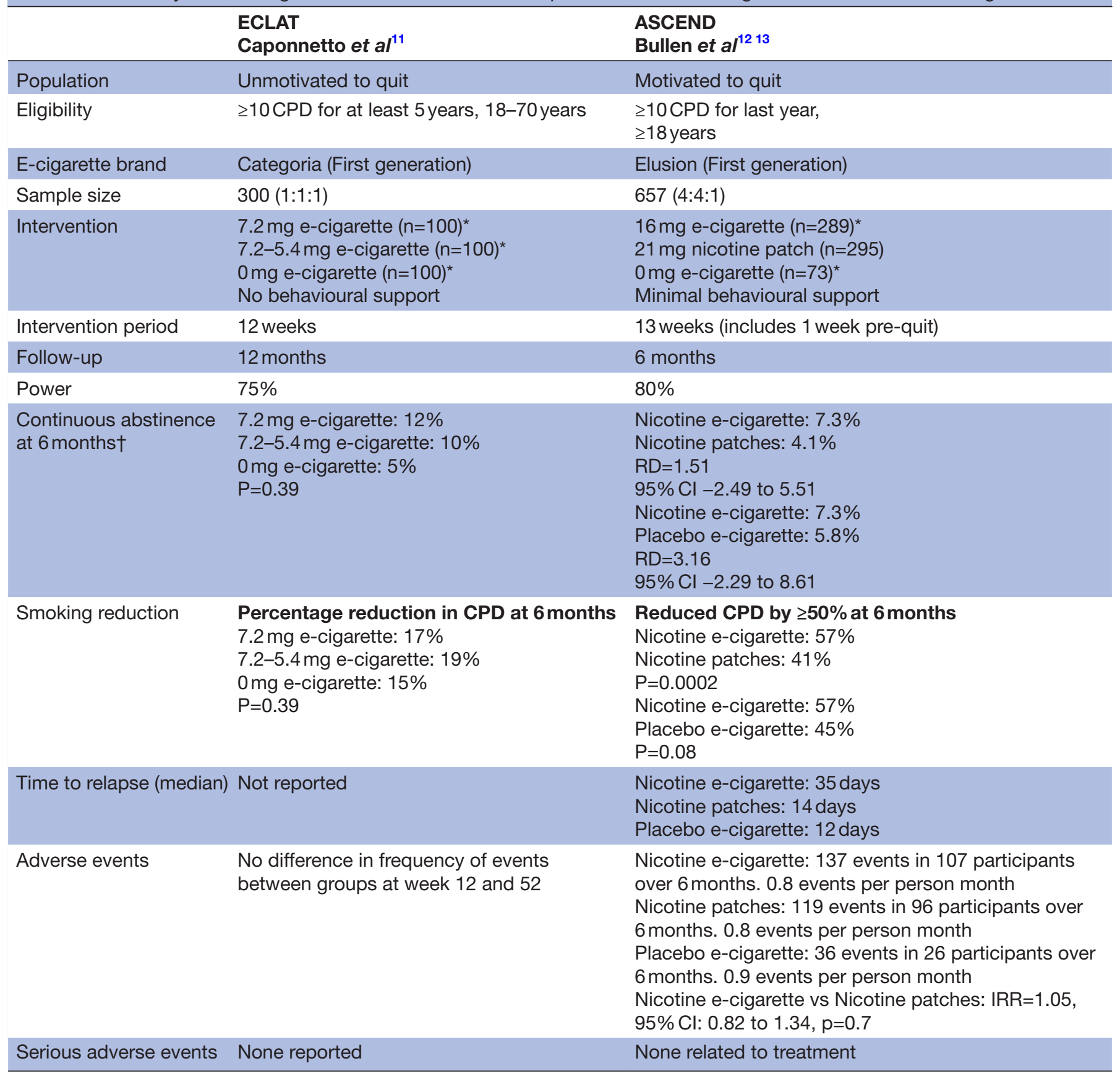

*Ad libutum use.

†Primary outcome.

$\mathrm{CPD}$, cigarettes per day; IRR, incidence rate ratio; $\mathrm{RD}$, risk difference.; $\mathrm{Cl}$, confidence interval

outcomes have been published on the use of e-cigarettes for smoking cessation (table 1$).{ }^{11-13}$

In New Zealand (NZ), nicotine is regulated as a medicine, except when delivered in tobacco smoke. Up until June 2018, it was illegal to sell an e-cigarette that contained nicotine or to make a cessation claim about e-cigarettes, because Medsafe (NZ's authority for licensing medicines) considered e-cigarettes a medicine if a cessation claim was made, or when supplied with nicotine. The case for maintaining the status quo in NZ (ie, only nicotine-free e-cigarettes available for sale) was that the efficacy of e-cigarettes is largely due to their behavioural replacement for conventional cigarettes. Indeed, some studies report a reduction in cravings to smoke with nicotine-free e-cigarettes, ${ }^{12} 13$ and point to some degree of support for cessation. Prior to June 2018, if an e-cigarette user in $\mathrm{NZ}$ wanted to have nicotine, they could combine the use of an e-cigarette with NRT. However, to date no trial has investigated the impact of combining NRT and e-cigarettes on smoking abstinence. There is good evidence 
that combining NRT products (eg, slow-acting nicotine patches combined with faster-acting oral products, such as lozenges, gum or mouth spray) is more effective than monotherapy alone, and as safe. ${ }^{14}$

In 2015 we received funding for a clinical trial to assess the effectiveness and safety of combining nicotine patches with e-cigarettes (with and without nicotine) on smoking abstinence at 6 months. Our primary hypothesis is that $21 \mathrm{mg}$ nicotine patches plus $18 \mathrm{mg} / \mathrm{mL}$ nicotine e-cigarettes will be more effective at helping smokers quit than $21 \mathrm{mg}$ nicotine patches plus nicotine-free e-cigarettes. Our secondary hypothesis is that a combination therapy (ie, $21 \mathrm{mg}$ nicotine patches plus $18 \mathrm{mg} / \mathrm{mL}$ nicotine e-cigarettes) will be more effective than monotherapy (ie, $21 \mathrm{mg}$ nicotine patches alone).

\section{METHODS AND ANALYSIS \\ Choice of design and intervention}

A three-arm, randomised-controlled, parallel group, superiority trial is used to answer the research question. While it is logical to include a fourth group (ie, placebo patches), it is unethical in NZ to deny a smoker access to a proven smoking cessation medication. For this reason, only an active nicotine patch is used. A $21 \mathrm{mg}$ (24hours) nicotine patch was selected as it is the standard patch strength used in NZ (Habitrol $21 \mathrm{mg}$ patch, Novartis Consumer Health Australasia Pty). A second generation e-cigarette starter kit (eVOD brand, 1.8 OHM: Kangertech, Shenzhen GuangDong, China) was chosen and purchased from NZVAPOR (https://www.nzvapor. $\mathrm{com} /$ ), with an $18 \mathrm{mg} / \mathrm{mL}$ nicotine strength. Each kit contains two batteries, two cartridges, two charging kits, one carry case and five atomisers. Participants can choose one of two tobacco e-juice flavours, based on the type of tobacco they usually smoke (ie, roll-your-own or factory-made: $38 \%$ of NZ smokers use roll-your-own tobacco exclusively $\left.{ }^{15}\right)$. The e-liquid $(60 / 40 \mathrm{PG} / \mathrm{VG}$ ratio) is sourced from Nicopharm, Australia (https://www. nicopharm.com.au/). The e-juice will be independently assessed to verify nicotine content is as labelled, and to check for contaminants. For the nicotine e-liquid, a variability of $\pm 10 \%$ nicotine concentration will be considered acceptable. Batch-to-batch variability of nicotine content in the e-liquid will also be assessed.

\section{Patient and public involvement}

Smokers and members of the public were not involved in the development of the research question, study design, recruitment or trial conduct. Choice of outcome measures was not directly informed by smokers' priorities, experience or preferences, nor has the burden of the intervention been assessed by smokers. However, the brand and type of e-cigarette, nicotine strength for the e-juice and choice of flavours was selected based on advice received from members of the NZ vaping retailer community. A summary of the study results will be posted/emailed to all trial participants.

\section{Study population}

People who smoke cigarettes (daily and non-daily), currently live in NZ, state that they are motivated to set a quit date within the next 2 weeks, and meet the eligibility criteria outlined below. Non-daily smokers are included for a number of reasons: (1) there is a drive to reach NZ's smokefree2025 goal; (2) there is limited research funding in NZ, so research efforts should endeavour to reach as many smokers as possible; (3) unpublished data from the NZ Health Survey show an increase in the number of non-daily smokers (from $7.7 \%$ in $2006 / 07$, to $9.3 \%$ in 2012/13); and (4) these non-daily smokers are less likely to receive cessation support. The risk versus benefit analysis of including this population in the trial considered the harms of continued smoking (and high likelihood of receiving no cessation support) versus the potential risk of exposure to higher than normal nicotine levels via the trial interventions (acknowledging that users will self-titrate).

\section{Eligibility criteria}

Participants will be eligible if they are at least 18 years of age, able to provide verbal consent, have access to a telephone and prepared to use the trial treatments. Only one person per household is eligible. There is no language restriction for participation in the trial, as translation services are available. Women who self-report that they are pregnant or breast feeding will be excluded from the trial, as will current users of NRT, people currently enrolled in another smoking cessation programme or cessation study, people who have used an e-cigarette for smoking cessation for more than 1 week any time in the last year or current users of non-nicotine based cessation therapies (eg, bupropion, clonidine, nortriptyline or varenicline). People are also ineligible if they have any contraindications to nicotine patches (ie, they have had a heart attack, stroke or severe angina within the previous 2 weeks, as per recommendations by the NZ Quitline) or e-cigarette (ie, they self-report a history of severe allergies and/or poorly controlled asthma). There are no other exclusion criteria-as a pragmatic trial all people who smoke are eligible for the trial, irrespective of their medical/psychiatric history.

\section{Recruitment}

Potential participants will be recruited via media advertising/social media and directed to contact the study centre at the University of Auckland's National Institute for Health Innovation by freephone, email, Facebook or through the study website.

\section{Randomisation, allocation concealment and sequence generation}

Potential participants will be phoned by a research assistant and provided with further information about the study. A two-step verbal consent process will be used (undertaken within the one call), where permission will be sought from participants to (1) undertake screening 
and (2) undertake randomisation. A copy of the patient information sheet and electronic consent form will be posted/emailed to participants for their records. After screening, baseline data will be collected and participants will be allocated to one of the three study groups in a 1:4:4 ratio $(21 \mathrm{mg}$ nicotine patch alone: $21 \mathrm{mg}$ nicotine patch plus $18 \mathrm{mg} / \mathrm{mL}$ nicotine e-cigarette: $21 \mathrm{mg}$ nicotine patch plus nicotine-free e-cigarette) using stratified block randomisation (block size of nine). Randomisation will be stratified by ethnicity (Māori, non-Māori) to ensure an equal balance in this key prognostic factor. The computer-generated randomisation sequence will be prepared by the study statistician.

\section{Blinding}

Participants and all research staff (except the project manager) are blinded to the nicotine content of the e-juice (the e-juice is stored in a brown bottle), until after data lock. The project manager is not involved in any data collection or interaction with trial participants. If required, the medical practitioner who reviews all adverse event reports may request that the participant's data be un-blinded. This un-blinding will be undertaken by the study statistician.

\section{Withdrawal}

If a participant voluntarily withdraws, no further data from the point of withdrawal will be collected. Should a participant require discontinuation of study treatment, or if they elect to cease taking treatment, data collection will continue as scheduled. If a participant discontinues treatment due to a serious adverse event, the participant will be followed until the event resolves or there is a return to a clinically acceptable medical status.

\section{Study interventions and procedures}

Participants will be randomised to one of three treatment arms:

- $21 \mathrm{mg}$ nicotine patch alone $(\mathrm{n}=201)$.

- $21 \mathrm{mg}$ nicotine patch plus $18 \mathrm{mg} / \mathrm{mL}$ nicotine e-cigarette $(n=804)$.

- $21 \mathrm{mg}$ nicotine patch plus nicotine-free e-cigarette $(\mathrm{n}=804)$.

Participants will receive 14 weeks of treatment, consisting of a 2 week pre-quit period to familiarise themselves with their allocated product(s) and 12 weeks post-quit treatment. ${ }^{12} 131617$ At the time of randomisation participants in all three arms will receive 10-15 min of telephone-based withdrawal-oriented behavioural support (based on cognitive behavioural therapy) and advice for using their allocated product. All three groups will also receive weekly withdrawal-oriented behavioural support telephone calls (10-15 min) for 6 weeks post-randomisation, delivered by trained smoking cessation advisers. Participants will have their full supply of free nicotine patches plus, if allocated, their free e-cigarette and e-juice (four $30 \mathrm{~mL}$ bottles) couriered to them.

\section{Pre-quit period}

At the time of randomisation, all participants will be advised to start using their nicotine patch (one per day) 2 weeks before their designated quit-date. During this 'pre-quit' period, those participants randomised to receive an e-cigarette will also be advised to start using their device ad libitum in order to familiarise themselves with use of the e-cigarette. ${ }^{12} 131617$ Participants will be provided with written instructions on how to assemble and use their e-cigarette, plus provided with a Web link to: (1) a NZ vaping industry designed document titled $A$ Beginners Guide to Vaping and (2) short on-line instruction videos hosted by a NZ-based on-line vaping retailer. This retailer will also provide a helpline number for participants to call should they need additional help or advice regarding use of the e-cigarette. The videos and helpline reflects 'real-world' support offered by the vaping community in NZ for naive e-cigarette users (with the exception that no face-to-face support will be offered, although participants are free to choose to visit a vape shop and/or talk with a vaper at any time during the trial if they wish).

\section{Intervention period}

All participants will be instructed to stop smoking tobacco cigarettes from their designated quit date forward, and continue with their allocated treatment for 12 weeks irrespective of any lapses back to smoking. All participants who have not quit by the end of follow-up will be provided with further cessation support within the context of publicly available cessation services in NZ.

\section{Baseline assessments}

The following baseline data will be collected via a phone interview with all participants:

- Demographics: date of birth, gender, ethnicity, self-reported height and weight and socio-economic position (based on education).

- Smoking history: frequency of smoking (daily or non-daily, and if the latter-with what frequency), age when started, number of cigarettes smoked per day (or when smoking, for non-daily smokers), years of smoking, number of previous attempts to give up in past 12 months (including the longest time they stayed quit and the method used), type of cigarettes smoked per day (eg, roll-your-own, factory-made), pack size and how long each pack lasts (for rollyour-own tobacco users), and whether they had tried to reduce the number of cigarettes smoked in the last 12 months.

- Level of cigarette dependence: measured using the Fagerström Test for Cigarette Dependence. ${ }^{18} 19$

- Other smoking-related information: self-rated chances of quitting measured on a scale from 1 to 5 where $1=$ unlikelyand 5 =highlylikely; smoking and e-cigarette use in the household; exposure to others who use e-cigarettes; smokefree home and car policies. 
- General health: self-reported shortness of breath, cough, asthma, chronic obstructive pulmonary disease (COPD), and current or history of mental health problems.

- The physical signs and symptoms associated with withdrawal: measured using the Mood and Physical Symptoms Scale (MPSS), ${ }^{20}$ including urge to smoke.

- Concomitant medication: information about types of medication currently used.

\section{Primary outcomes}

The primary outcome will be continuous abstinence to 6 months post quit-date, defined according to the Russell Standard (ie, self-report of smoking not more than five cigarettes from the quit date, supported by biochemical validation via exhaled carbon monoxide $(\mathrm{CO})$ measurement). ${ }^{21} \mathrm{CO}$ measurements will only be undertaken at the 6 month and 12 month time point, and will be undertaken face-to-face by a researcher or community-based cessation provider at a site convenient to the participant. A CO Monitor (Bedfont Smokerlyzer; Bedfont Scientific Ltd, Station Road, Harrietsham, Maidstone, Kent, ME17 1JA, England) will be used, with a reading of 9 ppm signifying abstinence. ${ }^{21}$

\section{Secondary outcomes}

Secondary outcomes will be assessed via phone interview with participants on their designated quit date, and at 1 , 3,6 and 12 months post quit-date (table 2).

- Continuous abstinence (1, 3 and 12 months): the proportion of participants who have stopped smoking, defined as self-report of smoking not more than five cigarettes from the quit date.

- Sevenday point prevalence (all time points): the proportion of participants who have stopped smoking, defined as self-report of having smoked no cigarettes (not even a puff) in the past 7 days.

- Change from baseline in the number of cigarettes smoked per day, or when smoking for non-daily smokers (all time points): if the participant is still smoking.

- Proportion of participants who have significantly reduced smoking (all time points): percentage reduction and the proportion who have reduced the number of cigarettes smoked per day (or when smoking for non-daily smokers) by at least $50 \%$ (in order to allow comparison with the ASCEND trial ${ }^{12}{ }^{13}$ ).

- Time to first relapse from quit date: defined as return to regular smoking (at least 5 cigarettes in the last 7 days).

- Use of any other smoking cessation methods (all time points).

- Medication compliance (quit day, 1 and 3 months): participants will be asked whether they used their allocated product(s), and if not, why not. Participants who did use their allocated products(s) will be asked when they last used them and how many days in the last week. Those allocated e-cigarettes will be asked how many millilitres of juice they use on a typical day (the EVod holds $2.2 \mathrm{ml}$ ).

- Crossover (all time points): participants in the patchonly group will be asked whether they accessed and used an e-cigarette (with or without nicotine) during the trial, and if so, at what time during the trial;

- Weight (3,6 and 12 months): self-reported.

- Change from baseline in the physical signs and symptoms associated with withdrawal (all time points): measured using the MPSS, ${ }^{20}$ including urge to smoke.

- Dual use (all time points): defined as daily use of both their allocated e-cigarette and usual cigarettes.

- General vaping questions (all time points): urge to vape, whether they changed devices and/or e-juice; whether they accessed any support for using their e-cigarette (and if so, where and how useful the support was); whether anyone they see at least once a week currently uses an e-cigarette (including whether this is someone they live with or not).

- Continuation of use (6 and 12 months): continued use of their allocated treatment after the end of the treatment period.

- General health (all time points): self-reported shortness of breath, cough, asthma, COPD, and mental health problems.

- Belief in ability to quit and stay quit (quit date and 1 month): measured on a scale from 1 to 5 where $1=$ unlikely and $5=$ highly likely.

- Identity (3 and 6 months): whether participants consider themselves a smoker, a smoker still trying to quit, an ex-smoker, and (in those allocated e-cigarettes) an ex-vaper, a vaper trying to quit smoking, a vaper trying to quit vaping, a vaper, other, or none of the above.

- Perception of their product (1, 3, 6 and 12 months): participants' views on use of their allocated treatment as a smoking cessation aid.

- Recommendations for use (1, 3,6 and 12 months): whether they would recommend their allocated treatment to another smoker who wanted to quit.

- Occurrence of specific side effects from product use (all time points): cough, nausea, dry mouth/throat, redness/swelling at patch site, dizziness, headache, vivid dreams, difficulty sleeping, dry skin, itchiness, other.

- Serious adverse events (all time points); Serious adverse events will be recorded and described as per International Conference on Harmonization-Good Clinical Practice (ICH-GCP) guidelines, and followed to resolution or stabilisation.

- Concomitant medication (all time points).

- Cost information: cost-per-quitter, cost-per-person reducing their daily cigarette consumption (or when smoking for non-daily smokers) and the incremental cost effectiveness ratio, if the intervention is indeed shown to be more effective than the comparison condition. The tobacco expenditure savings to individual smokers will also be calculated using data on 
Table 2 Details of follow-up

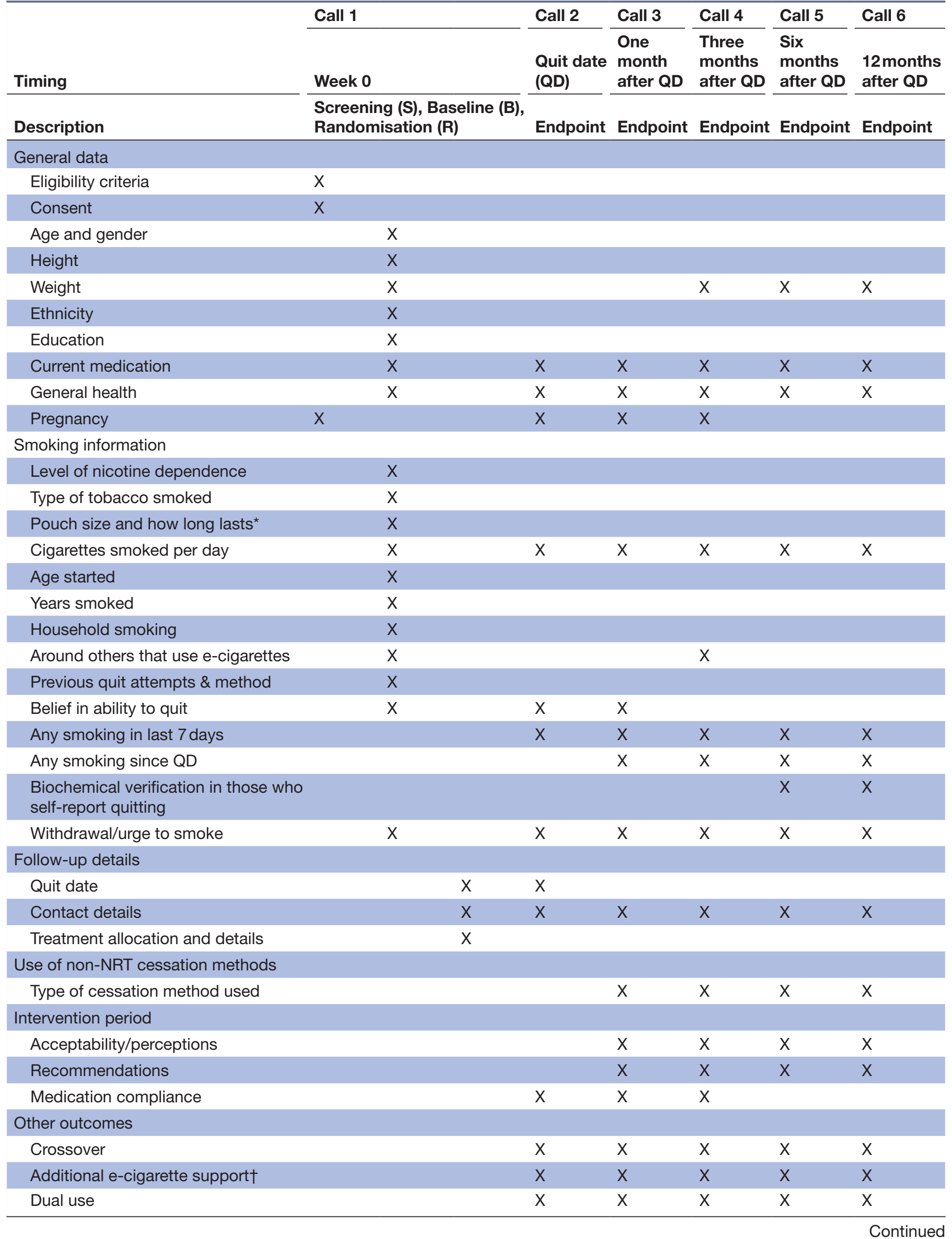


Table 2 Continued

\begin{tabular}{|c|c|c|c|c|c|c|}
\hline & Call 1 & Call 2 & Call 3 & Call 4 & Call 5 & Call 6 \\
\hline Timing & Week 0 & $\begin{array}{l}\text { Quit date } \\
\text { (QD) }\end{array}$ & $\begin{array}{l}\text { One } \\
\text { month } \\
\text { after QD }\end{array}$ & $\begin{array}{l}\text { Three } \\
\text { months } \\
\text { after QD }\end{array}$ & $\begin{array}{l}\text { Six } \\
\text { months } \\
\text { after QD }\end{array}$ & $\begin{array}{l}12 \text { months } \\
\text { after QD }\end{array}$ \\
\hline Description & $\begin{array}{l}\text { Screening (S), Baseline (B), } \\
\text { Randomisation (R) }\end{array}$ & Endpoint & Endpoint & Endpoint & Endpoint & Endpoint \\
\hline Cost & & $x$ & $x$ & $x$ & $x$ & $x$ \\
\hline Continuation of allocated treatment & & & & & $x$ & $x$ \\
\hline Side effects/serious adverse events & & $x$ & $x$ & $x$ & $x$ & $x$ \\
\hline Identity & & & & $\mathrm{X}$ & $\mathrm{x}$ & $x$ \\
\hline
\end{tabular}

*In people who smoke roll-your-own tobacco.

†ln those allocated e-cigarettes.

the amount smoked prior to quitting and the price of the particular products smoked.

\section{Sample size}

To detect an absolute difference of $8 \%$ in 6 month continuous abstinence rates between the $21 \mathrm{mg}$ nicotine patch +nicotine e-cigarette group and the $21 \mathrm{mg}$ nicotine patch +nicotine free e-cigarette group, 804 participants are needed in each group for $90 \%$ power (and 600 for $80 \%$ power). To detect an absolute difference of $15 \%$ in 6 month continuous abstinence rates between the $21 \mathrm{mg}$ nicotine patch group and the $21 \mathrm{mg}$ nicotine patch +nicotine e-cigarette group, 201 participants are needed in each group for $90 \%$ power (and 150 for $80 \%$ power). A total sample size of 1809 (804 in both e-cigarette groups and 201 in the nicotine patch group) is needed for $90 \%$ power, with $\mathrm{p}=0.05$ and adjusted for $20 \%$ loss to follow-up ${ }^{12}$ (figure 1).

A 6 month quit rate of $16 \%$ was assumed for the nicotine patch group, based on the average quit rate observed in the Cochrane review for nicotine patches vs placebo/no NRT control. ${ }^{14}$ We estimated a 6 month quit rate of $31 \%$ for the nicotine patch + nicotine e-cigarette group based on the quit rate observed in a trial $(n=239)$ comparing 'nicotine patches plus nicotine spray' against 'nicotine patches plus placebo spray'. ${ }^{22}$ A 6 month quit rate of $23 \%$ for the nicotine patch + nicotinefree e-cigarette group was assumed, based on a pragmatic trial $(n=1410)$ undertaken in NZ comparing use of NRT combined with very low nicotine cigarettes. ${ }^{23}$ Our previous experience of recruiting smokers from the community suggests recruitment will take 18 months. ${ }^{12}$

\section{Data management}

Members of the trial steering committee will provide trial oversight, with day-to-day management of the trial undertaken by the project manager, project coordinator and data manager. Study data will be collected by research assistants and directly entered into REDCap (Research Electronic Data Capture), a secure, Web-based application hosted at the University of Auckland and designed to support data capture for research studies. ${ }^{24}$ All data will be securely stored, regularly backed-up and retained for 10 years from data-lock. The study will be independently monitored after 10 participants have been randomised, at study close-out and twice during the trial. According to guidelines proposed by Ellenburg et al, ${ }^{25}$ a Data Safety and Monitoring Committee is not required..$^{25}$

\section{Statistical analysis}

Analyses will be performed using SAS (9.4) and R. ${ }^{26}$ No interim analyses are planned. Analysis will be carried out on an intention-to-treat basis (ie, all participants as originally allocated after randomisation will be analysed,

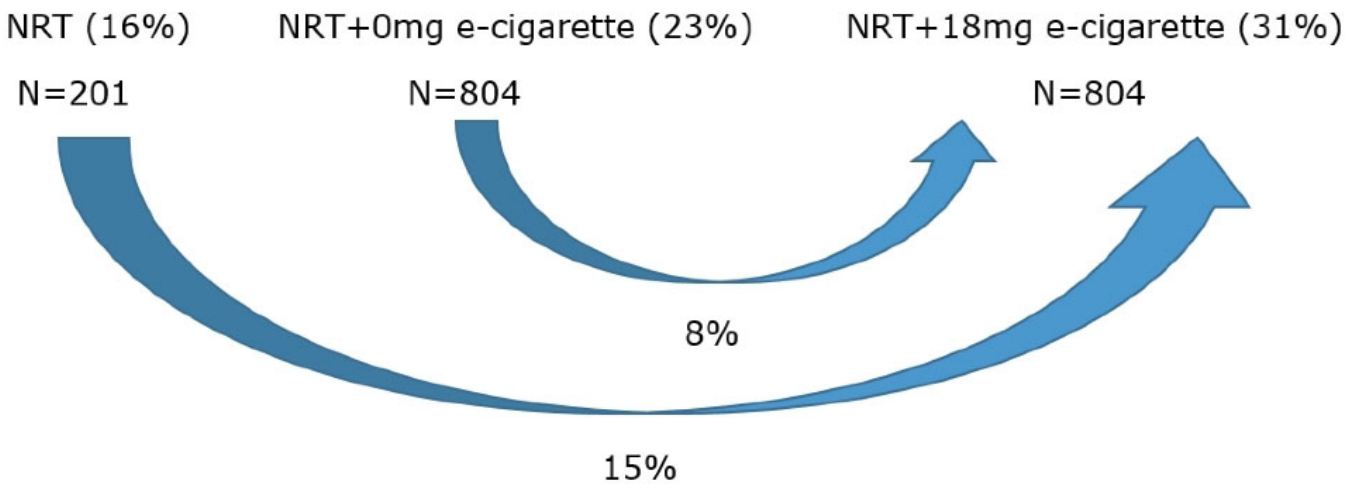

Figure 1 Estimated effect sizes for planned comparisons. 
and all participants lost to follow-up will be assumed to be smoking), with the quit rates, relative risks (RR), absolute risks and $95 \% \mathrm{CI}$ calculated for the primary and secondary comparison. Treatment groups will be compared using $\chi^{2}$ tests, with multiple logistic regression analysis adjusting for other variables as appropriate. Sensitivity analyses will be undertaken to determine the impact of using varying cut-offs for $\mathrm{CO}$ measurements (ie, at $\leq 3 \mathrm{ppm}, \leq 5 \mathrm{ppm}$ and $\leq 8 \mathrm{ppm}$ ) given lack of consensus about the best reading to use, and secondary analyses performed to correct overall cessation rates for discordance between reported and verified cessation. Sensitivity analyses will also be carried out to determine the effect of missing data. If the level of missing data is $>20 \%$, multiple imputation will be employed. ${ }^{27}$ Complete case analyses will be undertaken for the primary outcome, as well asper-protocol analyses where only those participants who completed the treatment originally allocated will be included (ie, participants with major protocol violations, such as cross-overs treatments, withdrawals and loss to follow-up will be excluded). The consistency of effects for pre-specified subgroups will be assessed using tests for heterogeneity. Subgroups will be based on age, sex, ethnicity, education, level of nicotine dependence, smoking frequency at baseline (daily/non-daily) and self-efficacy of quitting. Data related to smoking reduction will be reported separately for daily and non-daily smokers. A repeated measures model adjusted for baseline will be used to analyse change from baseline in cigarettes smoked per day (in non-abstainers), and change from baseline in weight and Body Mass Index. KaplanMeier curves, the log rank test and Cox proportional hazards regression analysis will be used to analyse time-torelapse. Serious adverse events will be defined according to the International Conference on Harmonization-Good Clinical Practice (ICH-GCP) E6 guidelines, categorised by the study doctor (masked to intervention product) as definitely, probably, possibly, unlikely or not related to the intervention, and coded by a medical coder (masked to intervention product) according to International Classication of Disease, tenth revision, Australian Modification (ICD-10 AM) (eighth edition). Events will be analysed by treatment group and association with study treatment. If the primary outcome of the trial is positive analyses will be undertaken to model the marginal cost-per-quitter, taking a health sector perspective. The tobacco expenditure savings to individual smokers will also be calculated (for those who quit and cut down) to give a more societal perspective on the financial benefits (especially to low-income smokers). For those participants who cut down their tobacco consumption by $\geq 50 \%$, the cost-perperson reducing their daily cigarette consumption will be calculated.

\section{ETHICS AND DISSEMINATION}

Participants are fully informed of their rights to withdraw, the risk/benefits of participating, the confidentiality of their data and that they may be eligible for compensation from the NZ Accident Compensation Corporation or private health/life insurance should they experience any injury as a result of participating in the trial. Approval from the Standing Committee on Therapeutic Trials was obtained on the 28 September 2015 for use of nicotine e-cigarettes. The trial dataset will be available from the corresponding author for use in any meta-analyses, on reasonable request. The dissemination plan includes national/international media

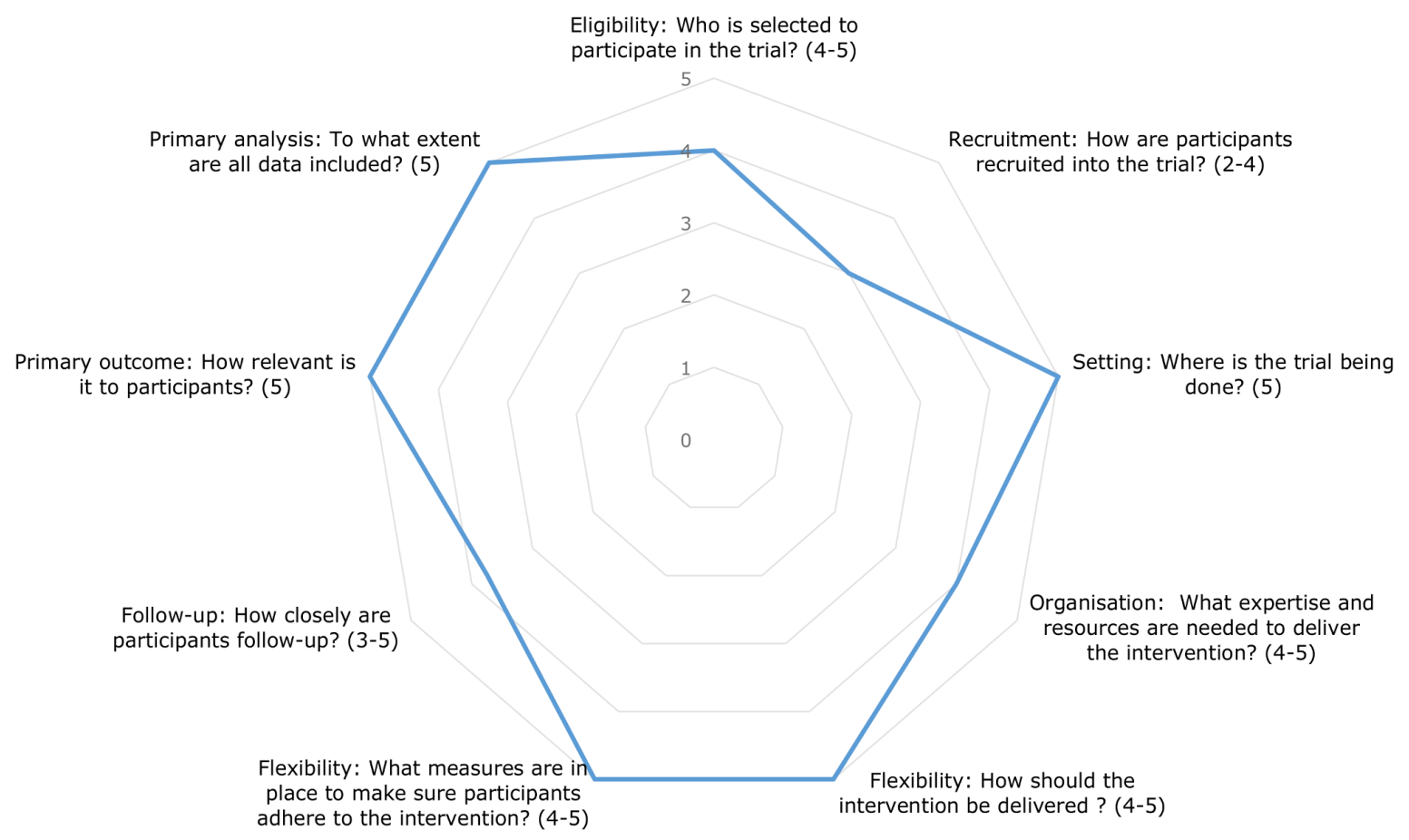

Figure 2 Design of the trial along the 'pragmatic to explanatory' continuum. 
coverage, publication in a high-impact peer-reviewed journal and oral presentations to relevant national/ international audiences.

\section{DISCUSSION}

One of the main limitations of clinical trials designed to prove whether e-cigarettes can help people quit smoking is that their findings are not generalisable, as the studied population is often very different to the general smoking population. For example, trial participants may be paid to participate in an effort to improve compliance and/ or retention, or subpopulations who are less likely to comply are excluded (such as people with mental health or alcohol use problems). This trial was designed to be as pragmatic as possible, with open eligibility and no patient payments (although trial medication/product was provided at no cost). This design will enable the findings to be more readily generalised to the unique tobacco control environment of NZ, where tobacco is expensive (NZ\$25.30, US\$18.20, €15.44 as 24 August 2017 for a pack of 20 cigarettes), tobacco advertising is banned, point-of-sale display bans are in effect and cessation support and medication (including combination NRT) is accessible and heavily subsidised. Despite these measures, in $201516 \%$ of the $\mathrm{NZ}$ adult population ( $\geq 15$ years) were current smokers (14\% daily), including $39 \%$ of Māori ${ }^{28}$ (indigenous NZers who comprise $15 \%$ of the population $^{29}$ ) and $25 \%$ of Pacific people ${ }^{28}$ (who comprise $5 \%$ of the population $\left.{ }^{29}\right)$. Within this environment, information on e-cigarette use by the population is limited. In 20112012, a survey of 480 adults ( $\geq 18$ years, smokers and recent quitters) found that $7 \%$ had ever purchased e-cigarettes. ${ }^{30}$ In 2016 , a survey of $3854 \mathrm{NZ}$ adults ( $>15$ years old, smokers and non-smokers) reported $17 \%$ had tried an e-cigarette, $3 \%$ were current users (defined as 'used at least daily, weekly or monthly'), and $1 \%$ were daily users of e-cigarettes. ${ }^{31}$

The pragmatic nature of the trial is highlighted by our use of a PRECIS-2 (PRagmatic-Explanatory Continuum Indicator Summary-2) wheel. $^{32}$ The wheel has nine spokes (or domains) that focus on each aspect of the trial, namely: eligibility, recruitment, setting, organisation, flexibility (delivery of the intervention), flexibility (adherence to the intervention), follow-up, primary outcome and primary analysis. Each domain is scored on a five-point Likert scale ranging from 1 'very explanatory' to 5 'very pragmatic'. More pragmatic trials have a larger wheel, while more explanatory trials have a smaller wheel. The tool also allows the reader to see that certain aspects of a trial may vary along the pragmatic-explanatory continuum. Five authors (NW, MV, TK, VP, CB) independently assessed the design according to the nine domains and the average scores for each domain are indicated on each spoke in figure 2 (with the range in brackets).

\section{Current status}

Recruitment started on 16 March 2016, with final data collection expected to be completed July 2018. This paper reports on protocol version 4.0, 10 February 2017. The protocol was amended in April 2017, driven by the need to shorten the interview time, reduce participant burden and ensure the trial can finish on budget and on time. The amendments involved removal of the 12 month assessment and several secondary outcomes, namely smokefree cars/homes (baseline); belief in ability to quit for good (1 month); MPSS and urge to smoke (all time-points); general vaping questions (all time-points); general health questions (quit date, 1 month); perceptions of their allocated product; and recommendations for use (one and 6 months). Details on the subset of participants that provided data on these removed secondary outcomes will be published. In June 2018 (4years after the trial was designed and funded), the NZ Ministry of Health legalised the sale and supply of nicotine e-cigarettes as consumer products.

Acknowledgements We thank members of the NZ vaping retailer community for their advice regarding the best type/brand of e-cigarette to use in this trial, plus the best nicotine strength and flavours for the e-juice. We also acknowledge the support of the funder, NZVAPOR, Nicopharm, Dr. Maciej Goniewicz, Angela Wadham and community smoking cessation providers throughout New Zealand.

Contributors Authorship follows the ICMJE guidelines. NW, VP, GL, ML and CB conceived the original idea for the trial, sought and obtained funding for the trial and wrote the study protocol. TK is the project manager responsible for the dayto-day running of the trial, while MV is the research fellow involved in the trial. VP will undertake all data analyses. This protocol paper was written by MV and NW with input from all co-authors. NW is guarantor for this paper. All authors read and approved the final manuscript.

Funding This trial is funded by a three year project grant from the Health Research Council of New Zealand (15/202). Approximately NZ\$600 000 (€350 000 or US $\$ 400000$ ) was available to run the trial. The e-cigarettes for this trial are being purchased directly from a New Zealand e-cigarette retailer - NZVAPOR. NZVAPOR are not involved in the design, conduct or analysis of the trial, but are providing on-line and phone support to participants regarding use of their allocated e-cigarettes. The e-cigarettes to be used in the trial and NZVAPOR (including the Managing Director) have no links with the tobacco industry. The e-juice for the trial is purchased directly from Nicopharm, Australia. The nicotine patches are supplied by the New Zealand Government via their contract with Novartis. Nicopharm and Novartis are not involved in the design, conduct or analysis of the trial and have no known links with the tobacco industry. Independent testing of the nicotine content of the e-juice will be undertaken by the NicoTar group at Roswell Park Cancer Institute, Buffalo, New York, USA.

Competing interests No authors have received financial support for the submitted work from any companies with a financial interest in the products under investigation. $\mathrm{CB}$ has received benefits in kind (accommodation expenses) from a manufacturer of smoking cessation medications. NW has provided consultancy to the manufacturers of smoking cessation medications, received honoraria for speaking at a research meeting and received benefits in kind and travel support from a manufacturer of smoking cessation medications (but over five year ago). NW, $\mathrm{CB}, \mathrm{MV}, \mathrm{GL}$ and VP are currently involved in a clinical trial in which varenicline and matching placebo are supplied by Pfizer under their Investigator-Initiated Research Program. MV has previously undertaken research supported by an unrestricted grant from Pfizer. None of the authors' spouses, partners or children have financial relationships that may be relevant to the submitted work. All authors have no nonfinancial interests that may be relevant to the submitted work.

Patient consent for publication Not required.

Ethics approval Ethics approval was obtained on the 16/09/2015 from the Northern A Health and Disability Ethics Committee (15/NTA/123).

Provenance and peer review Not commissioned; externally peer reviewed. 
Open access This is an open access article distributed in accordance with the Creative Commons Attribution Non Commercial (CC BY-NC 4.0) license, which permits others to distribute, remix, adapt, build upon this work non-commercially, and license their derivative works on different terms, provided the original work is properly cited, appropriate credit is given, any changes made indicated, and the use is non-commercial. See: http://creativecommons.org/licenses/by-nc/4.0/.

\section{REFERENCES}

1. Ferguson SG, Shiffman S. The relevance and treatment of cueinduced cravings in tobacco dependence. J Subst Abuse Treat 2009;36:235-43.

2. Przulj D, McRobbie $\mathrm{H}$. The effect of sensorimotor replacement on smoking cessation and craving. Open Addict J 2012;5:41-50.

3. Dawkins L, Turner J, Roberts A, et al. 'Vaping' profiles and preferences: an online survey of electronic cigarette users. Addiction 2013;108:1115-25.

4. Bullen $\mathrm{C}$, McRobbie $\mathrm{H}$, Thornley $\mathrm{S}$, et al. Effect of an electronic nicotine delivery device (e cigarette) on desire to smoke and withdrawal, user preferences and nicotine delivery: randomised cross-over trial. Tob Control 2010;19:98-103.

5. Glasser AM, Collins L, Pearson JL, et al. Overview of electronic nicotine delivery systems: a systematic review. Am J Prev Med 2017;52:e33-e66.

6. Hajek P, Etter JF, Benowitz N, et al. Electronic cigarettes: review of use, content, safety, effects on smokers and potential for harm and benefit. Addiction 2014;109:1801-10.

7. Goniewicz ML, Hajek P, McRobbie H. Nicotine content of electronic cigarettes, its release in vapour and its consistency across batches: regulatory implications. Addiction 2014;109:500-7.

8. Burstyn I. Peering through the mist: systematic review of what the chemistry of contaminants in electronic cigarettes tells us about health risks. BMC Public Health 2014;14:18.

9. Hecht SS, Carmella SG, Kotandeniya D, et al. Evaluation of toxicant and carcinogen metabolites in the urine of e-cigarette users versus cigarette smokers. Nicotine Tob Res 2015;17:704-9.

10. Chen J, Bullen C, Dirks K. A comparative health risk assessment of electronic cigarettes and conventional cigarettes. Int J Environ Res Public Health 2017;14:382.

11. Caponnetto P, Campagna D, Cibella F, et al. EffiCiency and Safety of an eLectronic cigAreTte (ECLAT) as tobacco cigarettes substitute: a prospective 12-month randomized control design study. PLoS One 2013;8:e66317.

12. Bullen $\mathrm{C}$, Howe $\mathrm{C}$, Laugesen $\mathrm{M}$, et al. Electronic cigarettes for smoking cessation: a randomised controlled trial. Lancet 2013;382:1629-37.

13. O'Brien B, Knight-West $\mathrm{O}$, Walker N, et al. E-cigarettes versus NRT for smoking reduction or cessation in people with mental illness: secondary analysis of data from the ASCEND trial. Tob Induc Dis 2015;13.

14. Stead LF, Perera R, Bullen C, et al. Nicotine replacement therapy for smoking cessation. Cochrane Database Syst Rev 2012;11:CD000146.
15. Young D, Wilson N, Borland R, et al. Prevalence, correlates of, and reasons for using roll-your-own tobacco in a high RYO use country: findings from the ITC New Zealand survey. Nicotine Tob Res 2010:12:1089-98.

16. Bullen $\mathrm{C}$, Knight-West $\mathrm{O}$. Issues in regulating e-cigarette clinical research. Clin Res Regul Aff 2015;32:1-5

17. Hajek P, Goniewicz ML, Phillips A, et al. Nicotine intake from electronic cigarettes on initial use and after 4 weeks of regular use. Nicotine Tob Res 2015;17:175-9.

18. Fagerstrom KO, Schneider NG. Measuring nicotine dependence: a review of the Fagerstrom Tolerance Questionnaire. J Behav Med 1989;12:159-82.

19. Heatherton TF, Kozlowski LT, Frecker RC, et al. The Fagerström Test for Nicotine Dependence: a revision of the Fagerström Tolerance Questionnaire. Br J Addict 1991;86:1119-27.

20. West R, Hajek P. Evaluation of the mood and physical symptoms scale (MPSS) to assess cigarette withdrawal. Psychopharmacology 2004;177(1-2):195-9.

21. West $R$, Hajek $P$, Stead L, et al. Outcome criteria in smoking cessation trials: proposal for a common standard. Addiction 2005;100:299-303.

22. Blondal T, Gudmundsson LJ, Olafsdottir I, et al. Nicotine nasal spray with nicotine patch for smoking cessation: randomised trial with six year follow up. BMJ 1999;318:285-8.

23. Walker N, Howe C, Bullen C, et al. The combined effect of very low nicotine content cigarettes, used as an adjunct to usual Quitline care (nicotine replacement therapy and behavioural support), on smoking cessation: a randomized controlled trial. Addiction 2012;107:1857-67.

24. Harris PA, Taylor R, Thielke R, et al. Research electronic data capture (REDCap)-a metadata-driven methodology and workflow process for providing translational research informatics support. J Biomed Inform 2009;42:377-81.

25. Ellenburg S, Fleming T, DeMets D. Data monitoring committees in clinical trials: a practical perspective. Chichester: John Wiley \& Sons, 2002

26. Bates D, Chambers JM, Dalgaard P, et al. The R Project for Statistica Computing Auckland. 1997. http://www.r-project.org (Accessed Mar 2018).

27. Hedeker D, Mermelstein RJ, Demirtas H. Analysis of binary outcomes with missing data: missing = smoking, last observation carried forward, and a little multiple imputation. Addiction 2007;102:1564-73

28. Ministry of Health. Annual Update of Key Results 2015/16: New Zealand Health Survey. Wellington: Ministry of Health, 2016.

29. Statistics New Zealand. New Zealand Census. Wellington: Statistics New Zealand, 2013.

30. Li J, Bullen C, Newcombe R, et al. The use and acceptability of electronic cigarettes among New Zealand smokers. N Z Med J 2013;126:ISSN 11758716.

31. Health Promotion Agency. Data release: Preliminary analysis on 2016 Health \& Lifestyle Survey electronic cigarettes questions. Wellington, New Zealand: Health Promotion Agency, 2017.

32. Loudon K, Treweek S, Sullivan F, et al. The PRECIS-2 tool: designing trials that are fit for purpose. BMJ 2015;350:h2147. 